\title{
(6) OPEN ACCESS \\ Methylation in the matrix metalloproteinase-2 gene is associated with cerebral ischemic stroke
}

\author{
Hsiu-Fen Lin, ${ }^{1,2}$ Edward Hsi, ${ }^{3,4}$ Ling-Chun Huang, ${ }^{1}$ Yi-Chu Liao, ${ }^{5,6}$ \\ Suh-Hang H Juo, ${ }^{3,4,7,8}$ Ruey-Tay Lin ${ }^{1,2}$
}

- Additional material is published online only. To view please visit the journal online (http://dx.doi.org/10. 1136/jim-2016-000277)

For numbered affiliations see end of article.

\section{Correspondence to}

Dr Ruey-Tay Lin, Department of Neurology, Kaohsiung

Medical University,

100 TzYou First Road,

Kaohsiung City 807, Taiwan; tay@kmu.edu.tw and

Dr. Suh-Hang Hank Juo, Institute of New Drug

Development, China Medical University, 91 Hsueh-Shih

Road, Taichung City 404,

Taiwan; hjuo@mail.cmu.edu. tw

Accepted 25 January 2017 Published Online First

13 February 2017

Copyright (C) 2016 American Federation for Medical Research

\section{ABSTRACT}

Matrix metalloproteinase-2 (MMP-2) is involved in the pathophysiology of stroke. Previous studies have shown that MMP-2 activity is increased in stroke; however, evidence of epigenetic regulation of the MMP-2 in stroke is still limited. We examined methylation of the MMP-2 promoter in patients with ischemic stroke. This study included 298 patients with ischemic stroke and 258 age-matched and sex-matched controls. MMP-2 promoter methylation levels were measured by pyrosequencing at eight potential cytosine-guanine (CpG) sites. Multivariate regression analysis was used to adjust for general stroke risk factors, and the specific effects of sex and stroke subtype were analysed. The methylation levels of MMP-2 in the peripheral blood of the patients with stroke were lower than controls in all eight CpG sites, especially at site 1, site 5 , site 7 , and site 8 (adjusted $p=0.036,0.002,0.021$, and 0.041 , respectively). In subgroup analysis by sex, a significant association was found only in men but not in women. When the stroke subtype was considered, men with small-vessel stroke had significantly lower methylation levels at all MMP-2 CpG sites than the controls (3.01\% vs 3.65\%, adjusted $p=0.018$ ). Although men with large-artery atherosclerosis stroke also had lower MMP-2 methylation levels, no significant difference was found $(3.25 \%$ vs $3.65 \%$, adjusted $p=0.253$ ). Demethylation of the MMP-2 promoter in patients with ischemic stroke was in a sex and stroke subtype-specific manners. These findings may add to the understanding of epigenetic modification of MMP-2 on ischemic stroke.

\section{INTRODUCTION}

Stroke is a leading cause of death and disability worldwide, and matrix metalloproteinases (MMPs), which are involved in extracellular matrix remodeling, have been reported to play a key role. ${ }^{12}$ The MMPs are a family of over 20 proteinases, in which gelatinase A (matrix metalloproteinase-2 (MMP-2)) is one of the main constitutive enzymes in the brain. MMP-2 has attracted increasing interest in the field of stroke pathophysiology, ${ }^{3} 4$ and it has been demonstrated that MMP-2 is involved in the atherosclerotic process. ${ }^{5}$ Higher levels of circulating MMP-2 have also been reported in cerebral ischemia, ${ }^{6}$ intracranial hemorrhage, ${ }^{7}$ and cerebral white matter hyperintensity. ${ }^{8}$ In

\section{Significance of this study}

\section{What is already known about this} subject?

- DNA methylation plays a major role in ischemic stroke.

- Matrix metalloproteinase-2 (MMP-2) activity is increased in cerebral stroke.

- The MMP-2 gene expression is regulated by epigenetic mechanisms, including methylation.

\section{What are the new findings?}

- This is the first study demonstrated lower MMP-2 methylation level in patients with ischemic stroke than controls.

- This effect was especially pronounced in men.

- With regards to the stroke subtype, MMP-2 methylation appeared to have more obvious influence in small-vessel stroke than large-vessel stroke.

How might these results change the focus of research or clinical practice?

- These findings may add to the understanding of epigenetic modification of MMP-2 on ischemic stroke.

addition, previous studies have suggested that MMP-2 plays multiple roles in ischemic stroke, including participating in the injury process in the early stage and contributing to recovery during the later stage. ${ }^{9}{ }^{10}$ In the human brain, levels of MMP-2 may remain high in ischemic lesions for up to several years after stroke. ${ }^{11}$ Taken together, these findings suggest that MMP-2 is a crucial regulatory marker for cerebral ischemic stroke.

Emerging evidence suggests that DNA methylation plays a major role in ischemic stroke. $^{12} 13$ In adult cells, DNA methylation takes place in a cytosine-guanine (CpG) dinucleotide context, also known as a $\mathrm{CpG}$ site, and in general, DNA methylation silences gene expression. ${ }^{14}$ It is known that the expression of the MMP-2 gene is regulated by epigenetic mechanisms, ${ }^{5} 15$ and it has also been demonstrated that a decrease in methylation of the MMP-2 gene can result in an increase in its 
expression in diseases including lung cancer, pterygium, and atherosclerosis. ${ }^{5} 1617$ Although previous studies have shown that circulating MMP-2 levels are increased in cerebral ischemia, ${ }^{6}$ no published study has investigated the relationship between MMP-2 methylation and ischemic stroke.

In this study, we evaluated methylation levels in the MMP-2 promoter using DNA from the peripheral blood of patients with ischemic stroke. Sex has been reported to be an important effect modifier regarding stroke risk factors and outcomes, ${ }^{18}$ and our previous study showed that global methylation had different effects on men and women with stroke. ${ }^{19}$ Therefore, we also investigated possible sex-specific effects on MMP-2 methylation in patients with ischemic stroke. As an increase in MMP-2 activity after ischemic stroke has only been reported in the smallvessel subtype of stroke, ${ }^{6}$ we also investigated MMP-2 methylation levels in patients with different stroke subtypes.

\section{MATERIALS AND METHODS \\ Subjects}

Stroke subjects

Two hundred and ninety-eight patients with ischemic stroke, aged between 40 and 80 years were selected from our previously reported patient cohort, ${ }^{20}$ all of whom were recruited from Kaohsiung Medical University Hospital and Taichung Veterans General Hospital in Taiwan. These patients were chosen as follows: (1) those with stroke in whom the first stroke episode occurred before 80 years of age; (2) these patient were then split into four age groups (40-50 years, 51-60 years, $61-70$ years, and $71-80$ years); and (3) an equal number of men and women were randomly selected from each age group. All of these patients were diagnosed with ischemic stroke according to the WHO criteria, ${ }^{21}$ which included laboratory examinations and cranial CT or MRI. Ischemic stroke was diagnosed when brain imaging showed acute infarction or revealed no evidence of hemorrhage. Stroke subtypes were classified according to the Trial of ORG 10172 in Acute Stroke Treatment (TOAST). ${ }^{22}$

\section{Control subjects}

The healthy controls were recruited from those who answered an advertisement at Kaohsiung Medical University Hospital and who reported no history of stroke or myocardial infarction. ${ }^{23}$ Two hundred and fifty-eight age-matched and sex-matched controls were recruited using random number tables created by SPSS statistical software (SPSS, Chicago, Illinois, USA). To minimise effects of age and gender, we selected an equal number of male and female cases and controls from each age group.

Data on socio-demographics, medical history of hyperlipidemia, hypertension, diabetes, and cigarette smoking were obtained for each participant. All the study subjects (including cases and controls) had no cancer history or peripheral basement membrane remodeling disease by selfreport. Glucose, triglycerides, and total cholesterol levels were tested from venous blood after fasting for at least 8 hours. Subjects with stroke had their blood collected in a subacute or chronic stroke stage. None of the samples were collected within 72 hours after acute stroke. A current smoker was defined as anyone who had smoked within the past 12 months of enrollment, and an ex-smoker was defined as someone who had stopped smoking for more than 1 year. Hypertension was diagnosed as systolic or diastolic blood pressure $\geq 140 / 90 \mathrm{~mm} \mathrm{Hg}$ or receiving antihypertensive medication. Diabetes was diagnosed as fasting blood glucose $\geq 126 \mathrm{mg} / \mathrm{dL}$ or receiving treatment for diabetes. Hypercholesterolemia was diagnosed as a serum level of total cholesterol $\geq 200 \mathrm{mg} / \mathrm{dL}$ or receiving lipid-lowering medications. The Institutional Review Boards of Kaohsiung Medical University Hospital and Taichung Veterans General Hospital approved this study, and the participants provided written informed consent.

\section{DNA extraction and MMP-2 methylation level measurement}

Genomic DNA was extracted using a commercially available DNA extraction kit (Gentra; Qiagen, Hilden, Germany). DNA samples were then treated with sodium bisulfate to convert unmethylated cytosine (C) to uracil and leave methylated $\mathrm{C}$ intact using an EpiTect Fast Bisulfite Kit (Qiagen) following the manufacturer's recommendations.

Using PyroMark Assay Design V.2.0 software (Qiagen), the MMP-2 methylation assay was designed to cover eight CpG sites in the promoter of the MMP-2 gene. The bisulfite-modified DNA was used to amplify the 178-bp product in the MMP-2 promoter area (figure 1) (primers are shown in online supplementary table). Quantitative MMP-2 methylation levels at the CpG sites were then measured by pyrosequencing (PyroMark Q24; Qiagen). Universal unmethylated and methylated DNA was run as the controls. Using PyroMark Q24 2.0.6 software (Qiagen), methylation quality was checked and quantified. The level of MMP-2 methylation was expressed as a percentage of methylated cytosine in methylated and unmethylated cytosines.

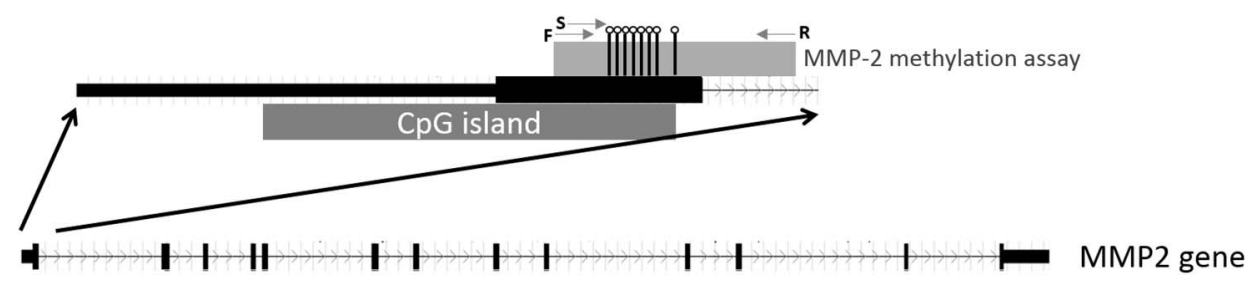

Figure 1 Schematic diagram for the design of the matrix metalloproteinase-2 methylation (MMP-2) assay. ${ }^{*} F, R$, and $S$ denote forward, reverse, and sequence primers, respectively. $\mathrm{CpG}$, cytosine-guanine sites. 


\section{Statistical analysis}

The $\chi^{2}$ test and Student's t-test were applied to analyse the characteristics of the study subjects. Associations between MMP-2 methylation level and ischemic stroke were analysed using the Student's t-test. Multivariate logistic regression analysis was then used to adjust for age, sex, smoking, hypertension, hypercholesterolemia, and diabetes. Data were also analysed by sex to assess sex-specific effects. The different effects of stroke subtypes were also assessed. SPSS software V.18.0 for Windows was used for all statistical analyses (SPSS), and a two-tailed $\mathrm{p}<0.05$ was considered to be statistically significant.

\section{RESULTS}

The demographic characteristics of the study subjects are presented in table 1 . With regards to risk factors for stroke, $76.2 \%$ of the patients with stroke had hypertension, $51.3 \%$ had diabetes, $50.3 \%$ had hypercholesterolemia, and $35.6 \%$ were current or ex-smokers. In the control group, $45.7 \%$ had hypertension, $10.9 \%$ had diabetic, $31.4 \%$ had hypercholesterolemia, and $15.1 \%$ were current or ever smokers. All risk factors were significantly different between the cases and controls as expected. With regards to stroke TOAST classification, $18.8 \%$ of the cases had large-artery atherosclerosis, $11.7 \%$ had cardioembolism, $45.0 \%$ had small-vessel occlusion, and $24.5 \%$ had an undetermined etiology.

The methylation levels in the peripheral blood at the eight potential MMP-2 promoter $\mathrm{CpG}$ sites are shown in table 2. In the MMP-2 CpG sites overall, the methylation levels were significantly lower among the patients with ischemic stroke compared with the controls in univariate analysis. After adjusting for traditional risk factors, the $\mathrm{p}$ values for $\mathrm{CpG}$ site 1 , site 5 , site 7 , and site 8 were still significant (adjusted $\mathrm{p}=0.036,0.002,0.021$, and 0.041, respectively). The average methylation level of all $\mathrm{CpG}$ sites was also significantly lower in the patients with ischemic stroke than in the controls (adjusted $\mathrm{p}=0.036$ ).

To assess sex-specific effects, we further stratified the participants by gender. Compared with the male controls, the male patients with ischemic stroke had lower MMP-2

\begin{tabular}{|c|c|c|c|}
\hline & $\begin{array}{l}\text { Stroke } \\
\mathrm{n}=298\end{array}$ & $\begin{array}{l}\text { Control } \\
\mathrm{n}=258\end{array}$ & p Value \\
\hline Age (years) & $61.5 \pm 10.3$ & $61.6 \pm 10.5$ & 0.901 \\
\hline Male (\%) & $147(49.3)$ & $121(46.9)$ & 0.610 \\
\hline Hypertension (\%) & $227(76.2)$ & $118(45.7)$ & $<0.001$ \\
\hline Diabetes (\%) & $153(51.3)$ & $28(10.9)$ & $<0.001$ \\
\hline Hypercholesterolemia (\%) & $150(50.3)$ & $81(31.4)$ & $<0.001$ \\
\hline Current and ex-smoker (\%) & $106(35.6)$ & 39 (15.1) & $<0.001$ \\
\hline \multicolumn{4}{|l|}{ Stroke subtype } \\
\hline Large-artery atherosclerotic & $56(18.8)$ & & \\
\hline Cardioembolism & $35(11.7)$ & & \\
\hline Small vessel & $134(45.0)$ & & \\
\hline Undetermined & 73 (24.5) & & \\
\hline
\end{tabular}

Data are shown as mean \pm SD for quantitative variables and $\mathrm{n}(\%)$ for qualitative variables.
Table 2 Association between ischemic stroke and matrix metalloproteinase-2 methylation level

\begin{tabular}{|c|c|c|c|c|}
\hline $\begin{array}{l}\text { CpG position/ } \\
\text { methylation \% }\end{array}$ & $\begin{array}{l}\text { Stroke } \\
\mathrm{n}=298 \\
\text { Mean } \pm \text { SD }\end{array}$ & $\begin{array}{l}\text { Control } \\
n=258 \\
\text { Mean } \pm \text { SD }\end{array}$ & $\begin{array}{l}\text { Crude } \\
p \text { value }\end{array}$ & $\begin{array}{l}\text { Adjusted } \\
\mathrm{p} \text { value }\end{array}$ \\
\hline Site 1 & $1.88 \pm 1.02$ & $2.13 \pm 1.11$ & 0.005 & 0.036 \\
\hline Site 2 & $3.75 \pm 1.97$ & $4.24 \pm 2.29$ & 0.006 & 0.052 \\
\hline Site 3 & $4.23 \pm 1.95$ & $4.66 \pm 2.08$ & 0.013 & 0.093 \\
\hline Site 4 & $3.59 \pm 1.84$ & $3.96 \pm 2.10$ & 0.028 & 0.103 \\
\hline Site 5 & $1.92 \pm 0.88$ & $2.25 \pm 1.20$ & $<0.001$ & 0.002 \\
\hline Site 6 & $3.43 \pm 1.78$ & $3.86 \pm 2.14$ & 0.009 & 0.098 \\
\hline Site 7 & $2.70 \pm 1.17$ & $3.02 \pm 1.41$ & 0.003 & 0.021 \\
\hline Site 8 & $4.46 \pm 2.23$ & $4.98 \pm 2.38$ & 0.009 & 0.041 \\
\hline Average of all sites & $3.24 \pm 1.52$ & $3.64 \pm 1.74$ & 0.004 & 0.036 \\
\hline
\end{tabular}

methylation levels at all $\mathrm{CpG}$ sites than the controls, and especially at $\mathrm{CpG}$ site 5 (adjusted $\mathrm{p}=0.011$ ) (table 3 ). While the methylation levels were also lower at all $\mathrm{CpG}$ sites in the female patients than in the female controls, none of the differences reached statistical significance.

Since previous studies have reported an association between MMP-2 plasma levels and the small-vessel stroke subtype rather than large-artery atherosclerosis, ${ }^{6}$ we further analysed MMP-2 methylation levels in men stratified by stroke subtype. Compared with the controls, the male patients with small-vessel stroke had a significantly lower average methylation level at all MMP-2 CpG sites than the controls $(3.01 \%$ vs $3.65 \%$, adjusted $\mathrm{p}=0.018)$ (figure 2 ). Although the male patients with large-artery atherosclerosis also had a lower MMP-2 methylation level than the controls, the difference was not statistically significant $(3.25 \%$ vs $3.65 \%$, adjusted $\mathrm{p}=0.253$ ).

\section{DISCUSSION}

In this study, we found a lower MMP-2 methylation level in the DNA from peripheral blood of patients with ischemic stroke than in controls with no history of stroke. This effect was especially pronounced in men. With regards to the stroke subtype, MMP-2 methylation appeared to have a more pronounced effect in patients with small-vessel stroke than large-vessel stroke, which is consistent with a previous study that reported an increased MMP-2 serum level in patients with small-vessel stroke. ${ }^{6}$ To the best of our knowledge, this is the first study to investigate the association between methylation in the MMP-2 promoter and cerebral ischemic stroke, and in particular with regards to sex and stroke subtype-specific effects. The findings of this study add to the current knowledge of the epigenetic modification of MMP-2 on ischemic stroke.

MMP-2 has been reported to play a dual role in ischemic stroke. ${ }^{9}{ }^{10}$ After a focal cerebral ischemic insult, the early pathological effect of MMP-2 is the disruption of the blood-brain barrier through degradation of the basal lamina, which may contribute to hemorrhagic transformation and eventually neuronal cell death. ${ }^{9}$ Conversely, 
Table 3 Association between ischemic stroke and matrix metalloproteinase-2 methylation level by sex

\begin{tabular}{|c|c|c|c|c|c|c|c|c|}
\hline \multirow[b]{2}{*}{$\begin{array}{l}\text { CpG position/ } \\
\text { methylation \% }\end{array}$} & \multicolumn{4}{|l|}{ Men } & \multicolumn{4}{|l|}{ Women } \\
\hline & $\begin{array}{l}\text { Stroke } \\
\mathrm{n}=147 \\
\text { Mean } \pm \text { SD }\end{array}$ & $\begin{array}{l}\text { Control } \\
n=121 \\
\text { Mean } \pm S D\end{array}$ & $\begin{array}{l}\text { Crude } \\
\text { p value }\end{array}$ & $\begin{array}{l}\text { Adjusted } \\
\mathrm{p} \text { value }\end{array}$ & $\begin{array}{l}\text { Stroke } \\
\mathrm{n}=151 \\
\text { Mean } \pm \text { SD }\end{array}$ & $\begin{array}{l}\text { Control } \\
n=137 \\
\text { Mean } \pm S D\end{array}$ & $\begin{array}{l}\text { Crude } \\
p \text { value }\end{array}$ & $\begin{array}{l}\text { Adjusted } \\
\mathrm{p} \text { value }\end{array}$ \\
\hline Site 1 & $1.81 \pm 0.98$ & $2.14 \pm 1.13$ & 0.012 & 0.064 & $1.94 \pm 1.06$ & $2.12 \pm 1.10$ & 0.153 & 0.253 \\
\hline Site 2 & $3.61 \pm 1.99$ & $4.21 \pm 2.34$ & 0.024 & 0.121 & $3.89 \pm 1.95$ & $4.27 \pm 2.25$ & 0.121 & 0.245 \\
\hline Site 3 & $4.08 \pm 1.97$ & $4.73 \pm 2.18$ & 0.011 & 0.135 & $4.38 \pm 1.94$ & $4.59 \pm 2.00$ & 0.354 & 0.453 \\
\hline Site 4 & $3.43 \pm 1.77$ & $3.95 \pm 2.24$ & 0.034 & 0.130 & $3.75 \pm 1.89$ & $3.97 \pm 1.98$ & 0.341 & 0.464 \\
\hline Site 5 & $1.82 \pm 0.83$ & $2.25 \pm 1.31$ & 0.001 & 0.011 & $2.02 \pm 0.91$ & $2.25 \pm 1.10$ & 0.051 & 0.061 \\
\hline Site 6 & $3.30 \pm 1.79$ & $3.92 \pm 2.33$ & 0.014 & 0.175 & $3.56 \pm 1.76$ & $3.82 \pm 1.97$ & 0.238 & 0.352 \\
\hline Site 7 & $2.62 \pm 1.15$ & $3.04 \pm 1.48$ & 0.010 & 0.059 & $2.77 \pm 1.19$ & $3.01 \pm 1.35$ & 0.109 & 0.210 \\
\hline Site 8 & $4.33 \pm 2.25$ & $4.88 \pm 2.45$ & 0.056 & 0.266 & $4.59 \pm 2.21$ & $5.06 \pm 2.32$ & 0.079 & 0.092 \\
\hline Average of all sites & $3.12 \pm 1.51$ & $3.65 \pm 1.87$ & 0.011 & 0.095 & $3.36 \pm 1.53$ & $3.64 \pm 1.61$ & 0.141 & 0.221 \\
\hline
\end{tabular}

Adjusted $p$ values were adjusted for age, hypertension, diabetes, hypercholesterolemia, and smoking status.

$\mathrm{CpG}$, cytosine-guanine sites.

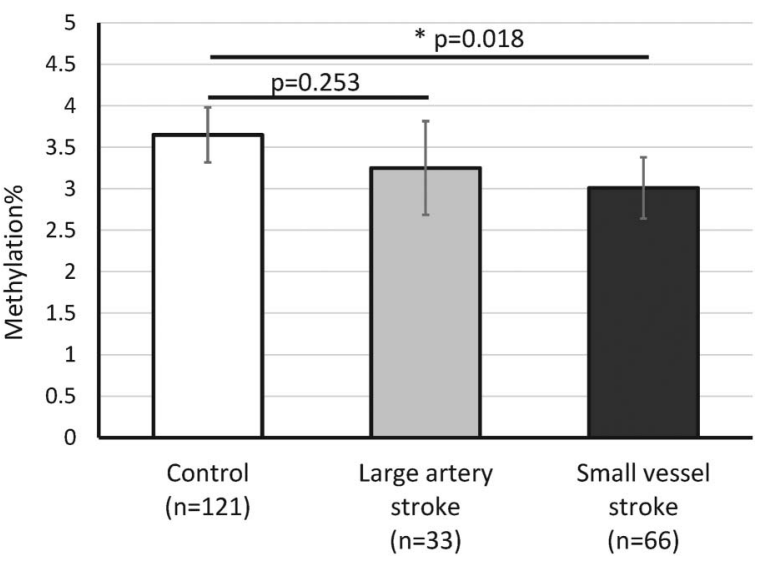

Figure 2 The association between promoter methylation level of matrix metalloproteinase-2 gene and stroke subtype in men.

MMP-2 also plays a role in endogenous repair, particularly in angiogenesis and in reestablishing cerebral blood flow during the poststroke recovery stage. ${ }^{10}$ Using gelatin zymography of human brain tissue, an increase in the activity of MMP-2 was observed in patients who died even several months after stroke. ${ }^{11}$ This prolonged elevation implies that MMP-2 is involved in recovery after a stroke. Furthermore, several studies have investigated the correlation between MMP-2 genetic polymorphisms and ischemic stroke, and the results showed that the patients with risk alleles had a 1.5-2.6-fold higher risk of ischemic stroke. $^{24-26}$ Taken together, these findings suggest that MMP-2 plays a crucial role in ischemic stroke.

Accumulating clinical and experimental evidence supports that DNA methylation is involved in the pathogenesis of ischemic stroke and that it is a risk factor for atherosclerosis, which is thought to be a major pathological change in stroke. ${ }^{27}$ In addition, several human studies have investigated associations between the level of DNA methylation and ischemic stroke. ${ }^{12}{ }^{19}$ Moreover, in our previous study, ${ }^{19}$ we found lower global methylation levels in long interspersed nucleotide element-1 (LINE-1) in male patients with stroke than in controls. In our recent study, we found that female patients with stroke had lower methylation levels of the estrogen receptor $\alpha$ gene than controls, especially in those with large-artery atherosclerosis and cardioembolic subtypes. ${ }^{12}$ The genetic expression of MMP-2 is known to be epigenetically regulated. ${ }^{5} 15$ Although several studies have explored MMP-2 genetic methylation status in several diseases, ${ }^{5} 1617$ no previous study has reported the effect of MMP-2 methylation in patients with ischemic stroke.

The clinical features of cerebral ischemic stroke including traditional risk factors and outcomes differ between men and women. ${ }^{18} 29$ Moreover, differences in plasma MMPs activity between men and women have been reported in human carotid plaque. ${ }^{30}$ In our previous study, we found that menopausal women carrying an MMP-9 risk allele were predisposed to stiffer arteries, whereas this risk allele had no such effect on premenopausal women. ${ }^{31}$ In addition, a previous study reported that the expression of MMP-2 is closely related to sex hormones. ${ }^{32}$ These biological mechanisms and findings from other studies further support our finding that MMP-2 methylation has a sexspecific effect in patients with ischemic stroke.

It has been reported that the effects of MMP-2 are limited to specific stroke subtypes. ${ }^{6}{ }^{24}$ Lucivero et $a l^{6}$ reported an increase in plasma MMP-2 level in patients with lacunar stroke, but not in those with large-artery atherosclerosis stroke. In addition, several MMP-2 polymorphisms were reported to be independent risk factors for small-vessel infarction in a Caucasian population, but not for other stroke subtypes. ${ }^{24} \mathrm{~A}$ recent study on a Chinese population also reported a significant association between MMP-2 genetic polymorphism and small-vessel disease. ${ }^{26}$ In the present study, a decrease in MMP-2 methylation level was only observed in the patients with small-vessel stroke, and not in those with large-artery atherosclerotic stroke, which is consistent with the previous results. Blood-brain barrier dysfunction has been reported to be a potentially important mechanism for small-vessel stroke, ${ }^{33}$ and MMP-2 has been reported to play a role in disruption of the blood-brain barrier after cerebral hypoperfusion. ${ }^{34}$ This could be a possible explanation for our finding of the association between MMP-2 demethylation and small-vessel stroke. 
There are limitations to this study. It is likely that some healthy controls might still have other hidden vascular diseases. Therefore, our results may need to be cautiously interpreted. Another limitation is that we do not have record on stroke severity for further analysis. It is always a concern when using a blood marker for a cerebral disease. In the current study, we measured MMP-2 methylation levels in peripheral leucocyte DNA, however, we did not have data to support a consistent level of methylation between peripheral blood and the brain, nor samples to measure serum MMP-2 levels. Although there is lack of comprehensive data, our results are consistent with MMP-2 activity reported in previous clinical stroke studies. ${ }^{6}$ Further studies are warranted to further investigate MMP-2 methylation in the context of ischemic stroke.

In summary, this study demonstrates that male patients with ischemic stroke have a lower MMP-2 methylation level, and that this effect is especially pronounced in patients with small-vessel stroke. Future studies on developing strategies to alter the activity of MMP-2 in patients with ischemic stroke should take sex and stroke subtypespecific effects into consideration.

\section{Author affiliations}

${ }^{1}$ Department of Neurology, Kaohsiung Medical University Hospital, Kaohsiung, Taiwan

${ }^{2}$ Department of Neurology, College of Medicine, Kaohsiung Medical University, Kaohsiung, Taiwan

${ }^{3}$ Department of Medical Research, Kaohsiung Medical University Hospital, Kaohsiung, Taiwan

${ }^{4}$ Department of Medical Research, China Medical University Hospital, Taichung, Taiwan

${ }^{5}$ Department of Neurology, Taipei Veterans General Hospital, Taipei, Taiwan

${ }^{6}$ Department of Neurology, National Yang-Ming University School of Medicine, Taipei, Taiwan

${ }^{7}$ Graduate Institute of Biomedical Sciences, China Medical University, Taichung, Taiwan

${ }^{8}$ Institute of New Drug Development, China Medical University, Taichung, Taiwan

Contributors H-FL, S-HHJ, R-TL conceived and designed the experiments. $\mathrm{Y}-\mathrm{CL}$, EH performed the experiments. H-FL, EH, L-CH analysed the data. H-FL, $\mathrm{S}-\mathrm{HHJ}, \mathrm{R}-\mathrm{TL}$ wrote the paper.

Funding This work was supported by grants from the Ministry of Science and Technology (Taiwan, R.O.C. 103-2314-B-037-029, 103-2314-B-037-027-MY2, and 104-2314-B-037-030), and Kaohsiung Medical University Hospital intramural grants (KMUH102-2R48).

Competing interests None declared.

\section{Patient consent Obtained.}

Ethics approval The Institutional Review Boards of Kaohsiung Medical University Hospital and Taichung Veterans General Hospital.

Provenance and peer review Not commissioned; externally peer reviewed.

Open Access This is an Open Access article distributed in accordance with the Creative Commons Attribution Non Commercial (CC BY-NC 4.0) license, which permits others to distribute, remix, adapt, build upon this work noncommercially, and license their derivative works on different terms, provided the original work is properly cited and the use is non-commercial. See: http:/l creativecommons.org/licenses/by-nc/4.0/

\section{REFERENCES}

1 Romanic AM, White RF, Arleth AJ, et al. Matrix metalloproteinase expression increases after cerebral focal ischemia in rats: inhibition of matrix metalloproteinase-9 reduces infarct size. Stroke 1998;29:1020-30.

2 Rosenberg GA, Navratil M, Barone F, et al. Proteolytic cascade enzymes increase in focal cerebral ischemia in rat. J Cereb Blood Flow Metab 1996:16:360-6.
3 Cunningham LA, Wetzel M, Rosenberg GA. Multiple roles for MMPs and TIMPs in cerebral ischemia. Glia 2005;50:329-39.

4 Yong VW, Power C, Forsyth P, et al. Metalloproteinases in biology and pathology of the nervous system. Nat Rev Neurosci 2001;2:502-11.

5 Chen KC, Wang YS, Hu CY, et al. OxLDL up-regulates microRNA-29b, leading to epigenetic modifications of MMP-2/MMP-9 genes: a novel mechanism for cardiovascular diseases. FASEB J 2011;25:1718-28.

6 Lucivero V, Prontera M, Mezzapesa DM, et al. Different roles of matrix metalloproteinases-2 and -9 after human ischaemic stroke. Neurol Sci 2007;28:165-70

7 Alvarez-Sabin J, Delgado P, Abilleira S, et al. Temporal profile of matrix metalloproteinases and their inhibitors after spontaneous intracerebral hemorrhage: relationship to clinical and radiological outcome. Stroke 2004;35:1316-22.

8 Corbin ZA, Rost NS, Lorenzano S, et al. White matter hyperintensity volume correlates with matrix metalloproteinase-2 in acute ischemic stroke. I Stroke Cerebrovasc Dis 2014;23:1300-6.

9 Heo JH, Lucero J, Abumiya T, et al. Matrix metalloproteinases increase very early during experimental focal cerebral ischemia. J Cereb Blood Flow Metab 1999;19:624-33

10 Silletti S, Kessler T, Goldberg J, et al. Disruption of matrix metalloproteinase 2 binding to integrin alpha vbeta 3 by an organic molecule inhibits angiogenesis and tumor growth in vivo. Proc Natl Acad Sci USA 2001;98:119-24

11 Clark AW, Krekoski CA, Bou SS, et al. Increased gelatinase A (MMP-2) and gelatinase B (MMP-9) activities in human brain after focal ischemia. Neurosci Lett 1997;238:53-6.

12 Lin HF, Hsi E, Liao YC, et al. Demethylation of circulating estrogen receptor alpha gene in cerebral ischemic stroke. PLOS ONE 2015;10:e0139608.

13 Schweizer S, Meisel A, Marschenz S. Epigenetic mechanisms in cerebral ischemia. J Cereb Blood Flow Metab 2013;33:1335-46.

14 Grummt I, Pikaard CS. Epigenetic silencing of RNA polymerase transcription. Nat Rev Mol Cell Biol 2003;4:641-9.

15 Pereira IT, Ramos EA, Costa ET, et al. Fibronectin affects transient MMP2 gene expression through DNA demethylation changes in non-invasive breast cancer cell lines. PLOS ONE 2014;9:e105806.

16 Moran A, Fernandez-Marcelo T, Carro J, et al. Methylation profiling in non-small cell lung cancer: clinical implications. Int I Oncol 2012;40:739-46.

17 Riau AK, Wong TT, Lan W, et al. Aberrant DNA methylation of matrix remodeling and cell adhesion related genes in pterygium. PLOS ONE 2011;6: e14687.

18 Niewada M, Kobayashi A, Sandercock PA, et al. Influence of gender on baseline features and clinical outcomes among 17,370 patients with confirmed ischaemic stroke in the international stroke trial. Neuroepidemiology 2005:24:123-8.

19 Lin RT, Hsi E, Lin HF, et al. LINE-1 methylation is associated with an increased risk of ischemic stroke in men. Curr Neurovasc Res 2014:11:4-9.

20 Lin HF, Tsai PC, Liao YC, et al. Chromosome 9p21 genetic variants are associated with myocardial infarction but not with ischemic stroke in a Taiwanese population. J Investig Med 2011;59:926-30.

21 The World Health Organization MONICA Project (monitoring trends and determinants in cardiovascular disease): a major international collaboration. WHO MONICA project principal i9nvestigators. I Clin Epidemiol 1988;41:105-14

22 Adams HP Jr, Bendixen BH, Kappelle LJ, et al. Classification of subtype of acute ischemic stroke. Definitions for use in a multicenter clinical trial. TOAST. Trial of Org 10172 in acute stroke treatment. Stroke 1993:24: 35-41.

23 Lin HF, Liu CK, Liao YC, et al. The risk of the metabolic syndrome on carotid thickness and stiffness: sex and age specific effects. Atherosclerosis 2010;210:155-9

24 Fatar $\mathrm{M}$, Stroick M, Steffens $M$, et al. Single-nucleotide polymorphisms of MMP-2 gene in stroke subtypes. Cerebrovasc Dis 2008;26:113-19.

25 Nie SW, Wang XF, Tang ZC. Correlations between MMP-2/MMP-9 promoter polymorphisms and ischemic stroke. Int I Clin Exp Med 2014;7:400-4.

26 Zhang $\mathrm{M}$, Zhu W, Yun W, et al. Correlation of matrix metalloproteinase-2 single nucleotide polymorphisms with the risk of small vessel disease (SVD). J Neurol Sci 2015:356:61-4

27 Chan GC, Fish JE, Mawji IA, et al. Epigenetic basis for the transcriptional hyporesponsiveness of the human inducible nitric oxide synthase gene in vascular endothelial cells. J Immunol 2005;175:3846-61.

28 Wang YS, Chou WW, Chen KC, et al. MicroRNA-152 mediates DNMT1-regulated DNA methylation in the estrogen receptor alpha gene. PLOS ONE 2012;7:e30635. 
29 Sheikh K, Bullock CM. Effect of measurement on sex difference in stroke mortality. Stroke 2007;38:1085-7.

30 Hellings WE, Pasterkamp G, Verhoeven BA, et al. Gender-associated differences in plaque phenotype of patients undergoing carotid endarterectomy. J Vasc Surg 2007;45:289-96; discussion 296-287.

31 Lin RT, Chen CH, Tsai PC, et al. Sex-specific effect of matrix metalloproteinase- 9 functional promoter polymorphism on carotid artery stiffness. Atherosclerosis 2012;223:416-20.
32 Natoli AK, Medley TL, Ahimastos AA, et al. Sex steroids modulate human aortic smooth muscle cell matrix protein deposition and matrix metalloproteinase expression. Hypertension 2005;46:1129-34.

33 Topakian R, Barrick TR, Howe FA, et al. Blood-brain barrier permeability is increased in normal-appearing white matter in patients with lacunar stroke and leucoaraiosis. J Neurol Neurosurg Psychiatr 2010;81:192-7.

34 Nakaji K, Ihara M, Takahashi C, et al. Matrix metalloproteinase-2 plays a critical role in the pathogenesis of white matter lesions after chronic cerebral hypoperfusion in rodents. Stroke 2006;37:2816-23. 